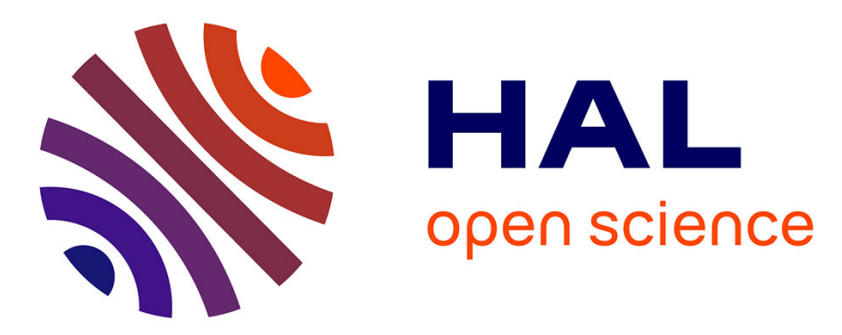

\title{
Accurate modelling of the carrier dynamics in nonlinear semiconductor nanoscale resonators
}

\author{
Gregory Moille, Sylvain Combrié, Alfredo de Rossi
}

\section{To cite this version:}

Gregory Moille, Sylvain Combrié, Alfredo de Rossi. Accurate modelling of the carrier dynamics in nonlinear semiconductor nanoscale resonators. Physical Review A, 2016, 94 (2), 10.1103/PhysRevA.94.023814 . hal-02935777

\section{HAL Id: hal-02935777 https://hal.science/hal-02935777}

Submitted on 10 Sep 2020

HAL is a multi-disciplinary open access archive for the deposit and dissemination of scientific research documents, whether they are published or not. The documents may come from teaching and research institutions in France or abroad, or from public or private research centers.
L'archive ouverte pluridisciplinaire HAL, est destinée au dépôt et à la diffusion de documents scientifiques de niveau recherche, publiés ou non, émanant des établissements d'enseignement et de recherche français ou étrangers, des laboratoires publics ou privés. 


\title{
Accurate modelling of the carrier dynamics in nonlinear semiconductor nanoscale resonators
}

\author{
Gregory Moille ${ }^{1,2}$, Sylvain Combrié ${ }$, and Alfredo De Rossi ${ }^{1 *}$ \\ ${ }^{1}$ Thales Research and Technology France, 1 avenue Augustin Fresnel, 91120 Palaiseau, France \\ 2 Institut d'Electronique Fondamentale, CNRS, Univ. Paris-Sud, \\ Université Paris-Saclay, Bâtiment 220, Rue André Ampère, F-91405 Orsay, France
}

(Dated: June 26, 2016)

\begin{abstract}
The Green's function formalism is used in order to model the diffusion of free carriers in nonlinear semiconductor nanoscale resonators. In combination with the time-dependent coupled mode equations, this leads to excellent agreement with measurements carried on a variety of samples and materials, using a minimal set of fitting parameters. The role of the geometry of the cavity is evidenced and the influence of linear and nonlinear absorption on the response of the resonator is discussed. This model can handle a broad range of phenomena: switching, self-pulsing, including resonant four-wave-mixing.
\end{abstract}

PACS numbers: 42.65.Pc,42.70.Qs, 78.20.Bh,78.66.Fd

\section{INTRODUCTION}

All-optical gates [1] modify the transmission of an optical signal with an optical control. This can be used to avoid the conversion into the electric domain, which, under some circumstances, would simplify the architecture of photonic integrated circuits. Nonlinear optical interactions are usually weak. However, it has been shown long ago that semiconductor cavities can produce a fast and strong nonlinear response, owing to the resonant enhancement and the substantial change of the refractive index induced by the excitation of free carriers [2]. An important point observed there is that the field confinement enhances the nonlinear response, but also results into a faster dynamics.

Nonlinear resonators in integrated photonic circuits have been proposed since the 90s, particularly in GaAs [3]. It was pointed out later that all-optical switching is feasible on a Silicon Photonics platform $[4,5]$. Modern fabrication techniques providing high-quality etching and sub-micron patterning, have enabled optical microcavities with close to diffraction-limited optical confinement and very low internal losses. The volume occupied by the optical field is here $V_{m} \approx(\lambda / n)^{3}$, implying that low input power translates into optical power density large enough to trigger nonlinear effects. More recently, alloptical gates with and femtojoule switching energy have been demonstrated owing to the combination of a very small optical resonator and optimized semiconductor material [6]. Moreover, a similar technology has been shown capable of optical read-only memory operation requiring a nanowatt power level. This should in principle allow scaling up to the Mbit optical storage capacity [7]. The integration of 25 individual gates on a single optical circuit has been reported very recently [8].

More subtle changes in the optical design have a remarkable and positive impact on the nonlinear dynamics of

*alfredo.derossi@thalesgroup.com nanoscale resonators [9]. The addition of quantum wells in the semiconductor layer [10] or conformal surface coating [11] have been successfully introduced to control the recovery dynamics.

The particularity of these nano-patterned semiconductor resonators is the role of geometry and, specifically, of the surface in determining how fast and how strong the nonlinear response is, because that is directly related to the generation, the diffusion and the recombination of free carriers. Interestingly, this has also been observed in micropillar resonators [12]. The dynamics of nonlinear resonators is extremely rich and is being actively studied. For instance, non-adiabatic dynamics [? ] and excitable [13] response has been observed, self pulsing has been predicted [14] and observed [15], multi-stability has been investigated in nonlinear coupled resonators [16] and, very recently, coherent effects have been evidenced in InP photonic crystal resonators [17].

A technical difficulty is that the carrier dynamics develops in a complicated geometry, which requires exact calculations of the diffusion equation. By itself, this is not an issue and has already been done [18]; however the connection to the Maxwell equations, describing the optical field in the structure, via direct coupling is very heavy computationally, because the time scales involved are very different. Thus, in the context of photonic nonlinear cavities, the diffusion equation has been solved only to provide an estimate of time constants for the rate equation. This approximation may not be satisfactory, for the reasons discussed in section IV.

We instead introduce a model where the exact calculation of the diffusion equation is reformulated using the Green's function formalism, which is allowed by the linearity of the diffusion equation in the ambipolar approximation, holding here. Thus, the time domain equation for the optical field can be indirectly but rigorously coupled to the diffusion equation and therefore this provides a more accurate model, only requiring a minimal set of fitting parameters, namely the surface recombination velocity. 


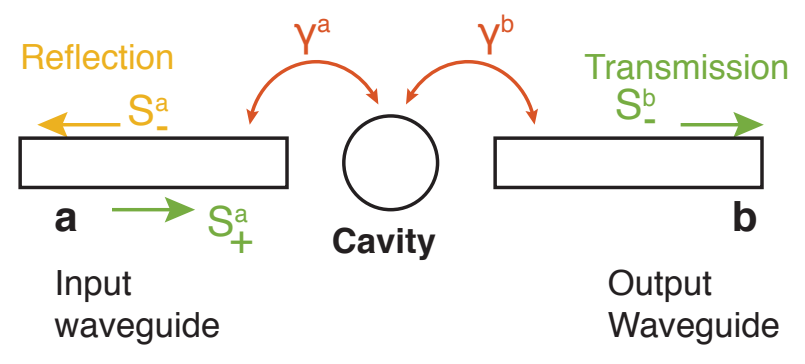

FIG. 1. Coupled Mode Theory: scheme representing a cavity coupled to 2 waveguides.

In section II we introduce the equations for the complex field amplitude describing an ensemble of coupled linear resonators and input/output waveguides and we discuss the main approximations. In section III we detail the nonlinear response and in section IV we describe the modelling of free carriers and the derivation of an effective carrier density response. The model is compared with experiments performed on different semiconductors in section V. Finally, we use the model to evidence the role of the geometry by comparing the response of different cavity design in section VI. We also compare the case of the excitation of free carriers through linear or nonlinear absorption in section VII.

\section{COUPLED MODE THEORY}

An ensemble of optical resonators coupled to waveguides is conveniently described by the Coupled Mode Theory in the time domain [19]. The basic assumption is that the optical field can be described in terms of normal modes in the waveguides and in the cavities. This approximation is correct as long as cavity modes are well confined, i.e. leakage can be treated as a small perturbation. While a more general situation is conveniently treated using quasi normal modes and suitable normalisation procedures [20,21], here the optical leakage is dominated by coupling into the cavity modes and the loaded $\mathrm{Q}$ factor is well above $10^{3}$. In single-mode cavities with mode size close to the diffraction limit, it can be shown that the energy density is about $Q$ times stronger than in the waveguide (see discussion in appendix A) and, therefore, the approximation is good enough.

\section{A. Formalism}

For the sake of simplicity, we shall consider the case of a single resonator (Figure. 1) coupled to two waveguides, serving as an input (port $a$ ) and an output (port $b$ ), with strengths $\gamma_{a}=\left|\kappa_{a}\right|^{2}$ and $\gamma_{b}=\left|\kappa_{b}\right|^{2}$ respectively. The complex envelopes of the incoming "+" and outgoing "-" waveguide modes are represented as vectors $\boldsymbol{S}_{\boldsymbol{i n}}=\left(S_{+, a}, S_{+, b}\right)^{\top}$ and $\boldsymbol{S}_{\boldsymbol{o u t}}=\left(S_{-, a}, S_{-, b}\right)^{\top}$ with subscript letters denoting the ports. The normal modes of the waveguides are normalized such that $\left|\boldsymbol{S}_{\boldsymbol{i n}}\right|^{2}=P_{\text {in }}$ and $\left|\boldsymbol{S}_{\text {out }}\right|^{2}=P_{\text {out }}$ representing the input and output powers (Fig. 1). The electric field in the cavity $\boldsymbol{E}(\boldsymbol{r}, t)=A(t) \boldsymbol{U}(\boldsymbol{r})$ is factorized into the complex envelope $A(t)$ and the normal mode $\boldsymbol{U}(\boldsymbol{r})$, which is normalized with the condition:

$$
\frac{1}{2} \int \varepsilon_{0} \varepsilon_{r}|\boldsymbol{U}(\boldsymbol{r})|^{2} \mathrm{~d} \boldsymbol{r}=1
$$

which defines the mode volume $V_{\bmod }^{-1}=\frac{\varepsilon_{0} \varepsilon_{r}}{2} \max \left(|\boldsymbol{U}|^{2}\right)$. Consequently, the energy in the cavity is $W_{\text {cav }}(t)=$ $|A(t)|^{2}$.

The motion equations [19] for the envelopes $A$, input $\boldsymbol{S}_{\text {in }}$ and outputs $\boldsymbol{S}_{\text {out }}$ are rewritten in the matrix formalism:

$$
\begin{aligned}
\partial_{t} A & =M A-\boldsymbol{R} \boldsymbol{S}_{i n} \\
\boldsymbol{S}_{\text {out }} & =\boldsymbol{Q} A+\boldsymbol{P} \boldsymbol{S}_{\text {in }}
\end{aligned}
$$

with $\boldsymbol{R}, \boldsymbol{Q}$ and $\boldsymbol{P}$ :

$$
\begin{aligned}
& \boldsymbol{R}=\left(\begin{array}{ll}
\kappa^{a} & \kappa^{b}
\end{array}\right) \\
& \boldsymbol{Q}=\left(\begin{array}{ll}
\kappa^{a} & \kappa^{b}
\end{array}\right)^{\top} \\
& \boldsymbol{P}=\left(\begin{array}{ll}
1 & 0 \\
0 & 1
\end{array}\right)
\end{aligned}
$$

In the linear limit, $M=-i \omega_{0}-\left(\Gamma_{0}+\gamma_{a}+\gamma_{b}\right) / 2$, with $\omega_{0} /(2 \pi)$ the frequency of the normal mode. With little loss of generality, we consider hereafter the situation where excitation is applied to port $a$, and therefore $S_{+, b}=0$.

This formalism can be generalized to handle an arbitrary number of cavities and waveguides. A very important assumption is that the resonators are weakly coupled together, such that super-modes are conveniently described in the tight-binding approximation. Recently it has been shown that in some case, e.g. chain of $\mathrm{PhC}$ cavities, some corrections are needed [22], which can be integrated in our model.

An interesting configuration consists of two resonators and two waveguides (Fig. 2). Then:

$$
\partial_{t} \boldsymbol{A}=\partial_{t}\left(\begin{array}{ll}
A_{1} & A_{2}
\end{array}\right)^{\boldsymbol{\top}}=\boldsymbol{M} \boldsymbol{A}-\boldsymbol{R} \boldsymbol{S}_{i n}
$$

Subscripts in $A_{k}$ relates to the two cavities and $M=M_{k l}$ is a $2 \times 2$ matrix:

$$
\begin{aligned}
& M_{k l}=i \omega_{k, 0}-\frac{1}{2}\left(\Gamma_{0, k}+\left|\kappa_{k, a}\right|^{2}+\left|\kappa_{k, b}\right|^{2}\right) \delta_{k l} \\
& M_{k l}=\left(i \mu-\kappa_{1, a} \overline{\kappa_{2, a}} \mathrm{e}^{-i \beta_{12}}-\kappa_{1, b} \overline{\kappa_{2, b}} / 2\right)\left(1-\delta_{k l}\right)
\end{aligned}
$$

$\Gamma_{0, k}$ is the photon decay rate, due to internal losses, $\mu$ the cavity mutual coupling, $\gamma_{k,(a, b)}$ the cavity to waveguide coupling, the overline denotes complex conjugation and $\delta_{k l}$ the Kronecker delta coefficient. $\boldsymbol{Q}, \boldsymbol{R}$ and $\boldsymbol{P}$ have the 


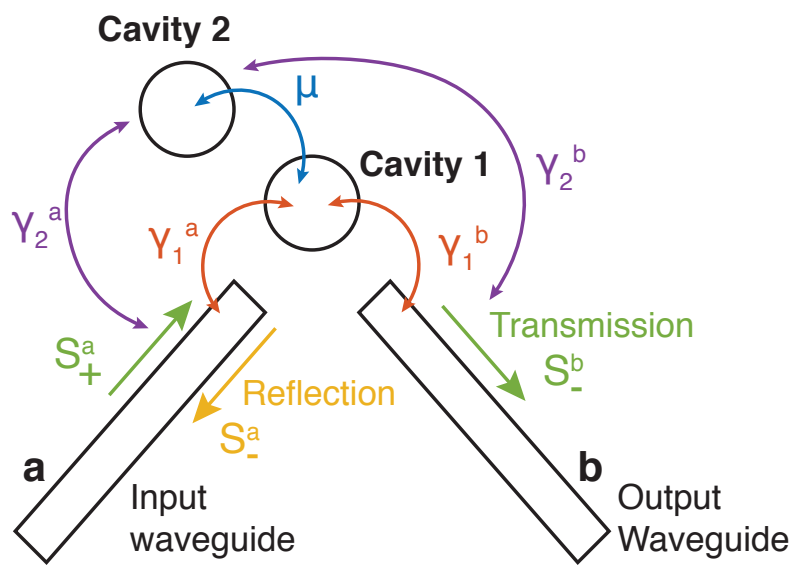

FIG. 2. Two coupled cavities and access waveguides and related parameters in the CMT model.

form:

$$
\begin{aligned}
\boldsymbol{R} & =\left(\begin{array}{cc}
\kappa_{1, a} & \kappa_{1, b} \\
\kappa_{2, a} \mathrm{e}^{-i \beta_{12}} & \kappa_{2, b} \mathrm{e}^{-i \beta_{12}}
\end{array}\right) \\
\boldsymbol{Q} & =\left(\begin{array}{ll}
\kappa_{1}^{a} & \kappa_{2}^{a} \mathrm{e}^{-i \beta_{12}} \\
\kappa_{1}^{b} & \kappa_{2}^{b} \mathrm{e}^{-i \beta_{12}}
\end{array}\right) \\
\boldsymbol{P} & =\left(\begin{array}{ll}
1 & 0 \\
0 & 1
\end{array}\right)
\end{aligned}
$$

The generalization to an arbitrary number of coupled cavities and input/output ports is straightforward and $\boldsymbol{R}, \boldsymbol{P}$ and $\boldsymbol{Q}$ are determined based on the energy conservation [19].

\section{B. Device design and comparison with linear measurements}

As an example, Fig. 3 represents the transmission spectra of $\mathrm{PhC}$ devices consisting of two waveguides and two coupled cavities made of different materials: InP, GaAs, and ALD-coated GaAs. The difference in the lineshapes are due to slightly different designs, as schematically represented in Fig. 2. The CMT reproduces the experimental transmission well with the fitted parameters listed in Appendix D. We point out that Fabry-Pérot modulation and other fast modulation of the spectra are absent here, owing to a design minimizing the length of the waveguides (and so coherent scattering) and to the use of adiabatic tapers to avoid reflection at their terminations. The design of the samples considered here are all based on two coupled $\mathrm{H}_{0}$ cavities [23], which are spaced by 3 lattice periods along the $M$ direction (Fig. 3d). In the case of the sample $a$, the position of the waveguides is such that the both cavities are coupled to them. The interference between the two paths results into a Fanolike lineshape (Fig.3a), which is the sample reported in ref. [24]. Samples b and c are instead based on a design such as only one of the two cavities is coupled to the waveguides. Consistently, Fano line-shape is absent in the transmission spectra.

The practical interest is that waveguides are on the same side of the sample, thus more convenient for experiments. The transmission minimum is actually a zero of the transmission spectra. The fact that they are not centered can be explained by a slight difference between the two cavity resonances (see Table III).

\section{NONLINEAR DYNAMICS}

Nonlinear effects induce a change in the complex permittivity $\varepsilon$ which we account for by allowing the complex resonance of each cavity $\omega_{k}=\omega_{k}^{0}+i \Gamma_{k}^{0} / 2$ to be corrected with the contribution $\Delta \tilde{\omega}=\Delta \omega_{k}+i \Delta \Gamma_{k} / 2$. Thus $\boldsymbol{M}$ becomes:

$$
M_{k k}^{N L}=i \Delta \omega_{k}\left(A_{k}\right)-\Delta \Gamma_{k}\left(A_{k}\right) / 2
$$

This choice implies that the nonlinear effect is local at the scale of the cavity. i.e. the nonlinearity in cavity $C_{i}$ only affects the other cavities $C_{j \neq i}$ indirectly, through the linear coupling coefficients $\gamma$ and $\mu$.

In the case of semiconductors, three dominant effects take place. The thermo-optic effect, which is slow (microsecond or longer) [25] and it not discussed here [26]. The nonlinear polarizability, i.e. the anharmonicity of the electronic potential, specifically the third order term $\chi^{(3)}$, inducing both a change in the real and imaginary part of the refractive index [27], as prescribed by KramersKronig relationship for physical transitions, e.g. absorption/emission. This phenomena is extremely fast when non-resonant, which is the case of semiconductors.

The term is expressed as:

$$
\left(\imath \Delta \omega_{k}-\frac{\Delta \Gamma_{k}}{2}\right)_{K e r r}=\left|A_{k}\right|^{2} c_{0}\left(\imath \omega n_{2}-\beta_{T P A} \frac{c_{0}}{2}\right) V_{\chi}^{-1}
$$

with $n_{2}$ the nonlinear index $\left(\Delta n=n_{2} I\right.$, I the irradiance), $\beta_{T P A}$ the two-photon absorption (defined from $\partial_{z} I=-\beta_{T P A} I^{2}$ ) and $V_{\chi}$ the nonlinear volume (appendix B).

We point out that this time-domain formulation of nonlinear responses also accounts for four-wave mixing, as long as the spectral domain of interest is narrow enough to validate the rotating wave approximation underlying the CMT.

The third effect is due to the excitation of free carriers, entailing a change of the refractive index. This consists of three contributions [28]: band-filling, free-carrier dispersion, and band shrinkage. We note that band shrinkage is negligible for GaAs or InP at Telecom wavelengths, and then will be neglected. In contrast to the nonlinear polarisation, the response of the refractive index to the optical excitation is not instantaneous, but follows the population of free carriers. It is convenient to define $N_{e f f}^{(k)}$ as the effective carrier density, i.e the carrier 

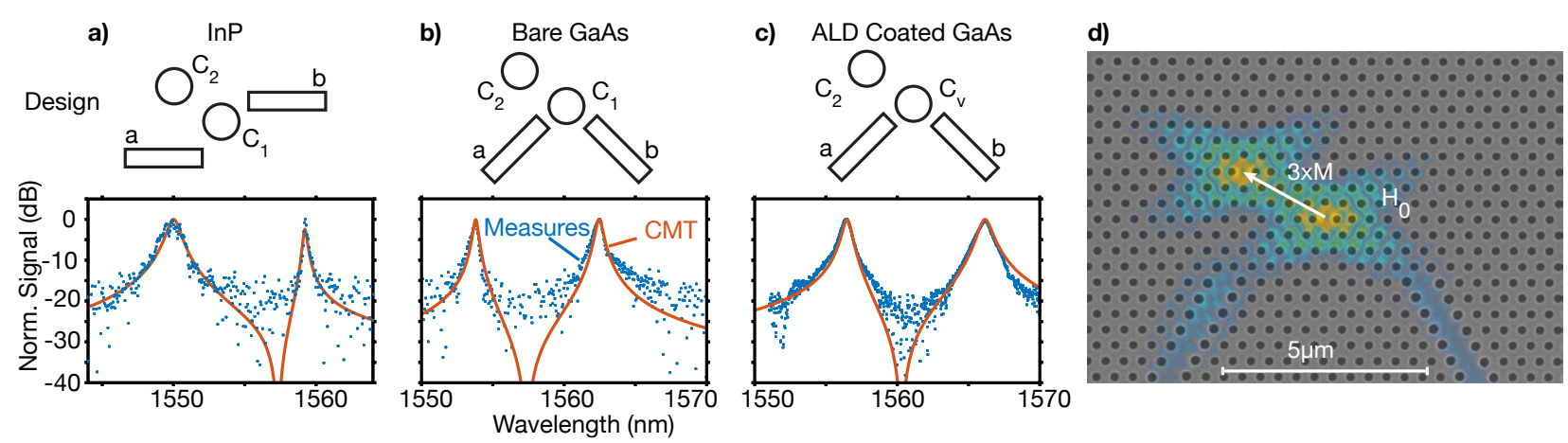

FIG. 3. Linear transmission measured (dots) and calculated (solid lines) with the CMT model for a) InP, b) GaAs and c) ALD-coated GaAs resonators. The corresponding design is sketched above; d) corresponding SEM image of the PhC structure with superimposed the mode profile.

density weighted by the spatial distribution of the cavity normal mode (see appendix C):

$$
N_{e f f}=\frac{1}{2} \varepsilon_{0} \int_{V} \varepsilon_{r} N(r, t)|\mathbf{U}(\mathbf{r})|^{2} \mathrm{~d} \mathbf{r}
$$

The corresponding contribution to the CMT model reads:

$$
\left(\imath \Delta \omega_{k}-\frac{\Delta \Gamma_{k}}{2}\right)_{F C}=\imath \frac{\omega}{n}\left(\frac{\partial \tilde{n}}{\partial N}\right)_{F C} N_{e f f}^{(k)}
$$

with the derivative representing the dependence of the complex index of refraction $\tilde{n}$ on the density of free carriers. Note that the definition of $N_{\text {eff }}$ already takes into account the connection between the refractive index and the frequency of the resonance (appendix $\mathrm{C}$ ).

\section{FREE CARRIERS DYNAMICS}

\section{A. Ambipolar model}

The modeling of ultrafast carrier dynamics has been broadly addressed in the context of semiconductor optical devices [29]. Here we use a more standard driftdiffusion model [30]. The justification of the underlying approximations is given in appendix C. In the absence of doping and external electric field, the evolution of photoexcited carriers in the domain $\Omega$ can be described in the ambipolar approximation of the drift-diffusion model, where electrons and holes have the same distribution, i.e. $N(\boldsymbol{r}, t)=P(\boldsymbol{r}, t):$

$$
\begin{array}{r}
\partial_{t} N-D_{a} \nabla^{2} N=G-\frac{N}{\tau} \quad \forall \quad r \in \Omega \\
D_{a} \nabla N \cdot \hat{n}=-S N \cdot \hat{n} \quad \forall \quad r \in \partial \Omega
\end{array}
$$

$D_{a}=\left(D_{n} \mu_{p}+D_{p} \mu_{n}\right) /\left(\mu_{p}+\mu_{n}\right)$ is the ambipolar diffusion coefficient, with $D_{n, p}$ and $\mu_{n, p}$ the diffusion coefficient and the mobility of electrons and holes respectively.
Considering two-photon absorption, the generation term is expressed as:

$$
G(\boldsymbol{r})=\beta_{T P A} \frac{c_{0}^{2} \varepsilon_{0}^{2}}{8 \hbar \omega} \varepsilon_{r}|\boldsymbol{U}(\boldsymbol{r})|^{4}|A|^{4}
$$

Note that this equation is based on the scalar approximation of the nonlinear tensor (appendix B). Integrating $G(\boldsymbol{r})$ we obtain the total generation rate, which is equal to the rate of absorbed photons, hence the equation:

$$
c_{0}^{2} \beta_{T P A} \frac{|A|^{4}}{2 \hbar \omega V_{\chi}}=-\frac{1}{2 \hbar \omega} \partial_{t}|A|^{2}
$$

consistently with equation 8 .

Carrier recombination has nonradiative and radiative contributions (see appendix $\mathrm{C}$ ), which are summarized here by a time constant $\tau$. In the specific case of photonic nanostructures, where the surface over volume ratio is $S / V=L_{s} \simeq 100 \mathrm{~nm}$ corresponding to about the average distance of the carriers from the nearest surface, thus surface recombination is extremely important. Considering the Shockley [31] approximation $\tau_{\text {eff }}^{-1}=\tau^{-1}+S / L_{s}$, the surface recombination may actually dominate the carrier lifetime, as shown in the case of GaAs photonic crystal cavities [32], as in GaAs $S$ might approach the saturation velocity [33] and, in general, is highly dependent on the material and the fabrication process.

This has two implications. First, radiative and nonradiative recombination in the bulk material plays a minor role, and, therefore, can very well approximated with a single time constant $\tau$. The second implication is that the validity of the rate equation model needs to be scrutinized in detail.

\section{B. Rate equations}

To clarify this point, let us assume that the carrier population decays following a time constant only determined by the dominant process, namely surface recombination. 
Then, a simple rate equation model could then represent the evolution of the excited carriers in the cavity, which is a very common approximation:

$$
\partial_{t} N_{e f f}=-\tau_{\text {eff }}^{-1} N_{e f f}+\frac{K_{T P A}}{V_{c}}|A|^{4}
$$

The generation term $K_{T P A}|A|^{4}=\int_{V} G(\boldsymbol{r})$ is obtained from eq. 13. A carrier volume $V_{c}$ is introduced empirically to obtain an effective generation density. It turns out that this approximation may not be satisfactory in the context of semiconductor nanocavities. Here, diffusion might also lead to an initial, extremely fast change of the carrier distribution seen by the cavity, i.e. $N_{\text {eff }}$. More interestingly, it has been observed experimentally that then the dynamics is slowed down in a variety of semiconductors: InP, GaInAsP and Silicon [5, 6, 34, 35]. This behaviour has been described with a multi-exponential decay, which is actually understood as resulting from the successive diffusion of photogenerated carriers to a larger reservoir with volume $V_{x}$ and population density $N_{x}$, until recombination takes place. This is readily seen using one or more auxiliary equations [35]:

$$
\begin{aligned}
& \partial_{t} N_{e f f}=-\gamma_{d i f f}\left(N_{e f f}-N_{x}\right)+\frac{K_{T P A}}{V_{c}}|A(t)|^{4} \\
& \partial_{t} N_{x}=-\tau_{\text {eff }}^{-1} N_{e f f}+\gamma_{d i f f} \frac{V_{c}}{V_{x}}\left(N_{e f f}-N_{x}\right)
\end{aligned}
$$

Accordingly, an auxiliary carrier diffusion rate $\gamma_{d i f f}$ is introduced. If this approach can represent the multiexponential decay observed experimentally, it is still not satisfactory for several reasons: first, the mode volume and the diffusion rate $\gamma_{d i f f}$ are not well-defined, and can only be used as a fitting parameters to coarsely model the diffusion process; second, more than one auxiliary equations may be necessary, as pointed out by $\mathrm{Yu}$ et al. for instance [36], therefore mechanically increasing the number of fitting parameters.

Actually, and this is a key point of this paper, the rate equation formalism, implying exponential decaying dynamics, is not the best choice for modelling a diffusion process. The reason of the failure of the exponential fit emerges when solving the diffusion equation analytically in the case of a gaussian initial spatial distribution and radial symmetry, namely $N(r, t)$. It is found that the spatial maximum of $N$ decays as $t^{-1}$. Although in our case the geometry is way more complex and the diffusion equation requires a numerical solution, this suggests a non-exponential decay of the carrier density (not the total population) when diffusion is important.

In this paper we introduce a major change in the model, consisting in replacing the rate equation with a more suitable representation of the diffusion dynamics. Moreover, since in semiconductor nanostructures this dynamics is essentially non local, as surface recombination and diffusion dominate, this demands an accurate calculation of carrier dynamics in the actual geometry. This is achieved in several steps, and will be detailed here below.

\section{Green's function formalism}

The diffusion equation (eq. 11), resulting from the ambipolar approximation, is a linear, partial derivatives equation; consequently, the temporal and spatial distribution of the free carriers can be related to the generation term (eq. 12) through a Green function $K\left(\boldsymbol{r}, \boldsymbol{r}^{\prime} ; \Delta \tau\right)$ :

$$
N(\boldsymbol{r}, t)=\int_{-\infty}^{t} \int K\left(\boldsymbol{r}, \boldsymbol{r}^{\prime} ; t-\tau\right) G\left(\boldsymbol{r}^{\prime}, \tau\right) \mathrm{d} \boldsymbol{r}^{\prime} \mathrm{d} \tau
$$

An interesting similarity appears with photo-reflectance measurements, where the time and spatial evolution of the temperature, following the excitation with a localized laser spot, are modelled through the Green's function formalism [? ? ]. There, the geometry allows the use of an analytic expression of the Green's function, which considerably eases the calculations. The geometry of PhC cavities, however, is such that the numerical calculation is unavoidable, which is an important point of this work. As a matter of fact, the calculation of the general Green's function is not necessary here, as the excitation (eq. 12) is a separable function in the form $G(\boldsymbol{r}, \tau)=\psi(\boldsymbol{r}) f(\tau)$. Therefore, the space integral in eq. 16 can be carried out to yield a simpler integral form:

$$
N(\boldsymbol{r}, t)=\int_{-\infty}^{t} \tilde{K}(\boldsymbol{r} ; t-\tau)|A(\tau)|^{4} \mathrm{~d} \tau
$$

with the kernel resulting from the above equations:

$$
\tilde{K}(\boldsymbol{r} ; \tau)=\beta_{T P A} \frac{c_{0}^{2} \varepsilon_{0}^{2}}{8 \hbar \omega} \varepsilon_{r} \int_{V} K\left(\boldsymbol{r}, \boldsymbol{r}^{\prime} ; \tau\right)|\boldsymbol{U}(\boldsymbol{r})|^{4} \mathrm{~d} \boldsymbol{r}^{\prime}
$$

The integral is carried out within the volume occupied by semiconductor, denoted by $V$. Note that $\tilde{K}(\boldsymbol{r} ; \tau)=0$ when $\tau<0$, because of causality.

The numerical calculation of the kernel is straightforward. Let us consider the Dirac excitation for eq. 17, namely in the form $|A(t)|^{4}=\left|A_{0}\right|^{4} \Delta t \delta(t)$ ( $\Delta t$ being an arbitrary small time interval). Using eq. 12 , this leads to the corresponding initial condition for eq. 11:

$$
N\left(\boldsymbol{r}, 0^{+}\right)=G\left(\boldsymbol{r}, 0^{+}\right) \Delta t
$$

This represents the distribution of carriers at $t=0^{+}$, after the Dirac impulse and before any diffusion process takes place. The equation 11 is then solved using a time domain finite element technique and the kernel is obtained through:

$$
N(\boldsymbol{r}, t)=\tilde{K}(\boldsymbol{r}, t)\left|A_{0}\right|^{4} \Delta t
$$

Let us now consider the projection of the carrier distribution on the optical mode, namely the effective carrier concentration introduced in section III. A new integral equation, relating $N_{\text {eff }}$ to the excitation $A(t)$, is obtained by combining eq. 9 with eq. 17 and changing the order of the integrals.

$$
N_{\text {eff }}(t)=\int_{-\infty}^{t} h_{0}(t-\tau)|A(\tau)|^{4} \mathrm{~d} \tau
$$


In the end, the Green's function formalism reduces to an explicit integral equation where the dynamics is contained in a time-dependent function $h_{0}(t-\tau)$, which, consistently with equations 9,17 and 21 , is defined as:

$$
h_{0}(\tau)=\frac{1}{2} \varepsilon_{0} \int \varepsilon_{r} \tilde{K}(\boldsymbol{r} ; \tau)|\mathbf{U}(\mathbf{r})|^{\mathbf{2}} \mathrm{d} \mathbf{r}
$$

In the context of signal theory, $h_{0}$ is referred to as an impulse response function. Hereafter, it will be referred to it as effective carrier density response function. Its initial value, which also corresponds to its maximum, is calculated by inserting eq. 18 into eq. 22 , which does not need solving the diffusion equation:

$$
h_{0}\left(0^{+}\right)=\frac{\beta_{T P A} c_{0}^{2} \varepsilon_{0}^{3}}{16 \hbar \omega} \int_{V} \varepsilon_{r}^{2}|\boldsymbol{U}(\boldsymbol{r})|^{6} \mathrm{~d} r^{3}=\frac{\beta_{T P A} c_{0}^{2}}{2 \hbar \omega} V_{3 P A}^{-2}
$$

Thus, $h_{0}\left(0^{+}\right)$, hence the maximum of effective carrier density, only depends on the field of the cavity mode $\boldsymbol{U}$. Interestingly, the equation above defines a higher order nonlinear interaction volume, $V_{3 P A}$, which is related to the Three Photon Absorption (3PA).

We note that these formulas are valid for the particular case of two-photons absorption. Linear absorption will be discussed in section VII.

As an example, $h_{0}$ is calculated for a $\mathrm{H}_{0}$ photonic crystal resonator (Fig. 4), depending on the surface recombination velocity $S$. First, the comparison between the spatially averaged kernel $\kappa(t)=\int_{V} K(\boldsymbol{r}, t) \mathrm{d} r$ (or, equivalently, the total population of of excited carriers) and $h_{0}$ reveals the role of the diffusion. In fact $h_{0}$ decays much faster than the total population of the carriers, as first pointed out in the context of Silicon $\mathrm{PhC}$ cavities [18]. This is also apparent in the false-color maps. Here, the distribution of carriers, represented by $\tilde{K}(\boldsymbol{r}, t)$ normalized by its maximum at $t=0$, evolves very differently, depending on the surface recombination.

The role of the diffusion becomes less important as the recombination $S$ increases, which is the case of GaAs nanostructures $[32,37,38]$, inherently related to its large density of defects at midgap [33]. It must be pointed out that the surface recombination is assumed to be identical at any interface, namely at the holes and at the top and bottom side of the slab. This might not be true, as different surfaces are exposed to different fabrication process steps. However, discriminating the values of $S$ depending on the surface is far from obvious.

In conclusion, given a geometry, the resonator material, and the surface recombination velocity, a unique effective carrier density response function $h_{0}$ is enough for modelling accurately the nonlinear dynamics of the resonator, including diffusion effects.

\section{MEASUREMENTS}

Our model is used to reproduce the dynamical response of $\mathrm{PhC}$ nanocavities made of different materials (InP and
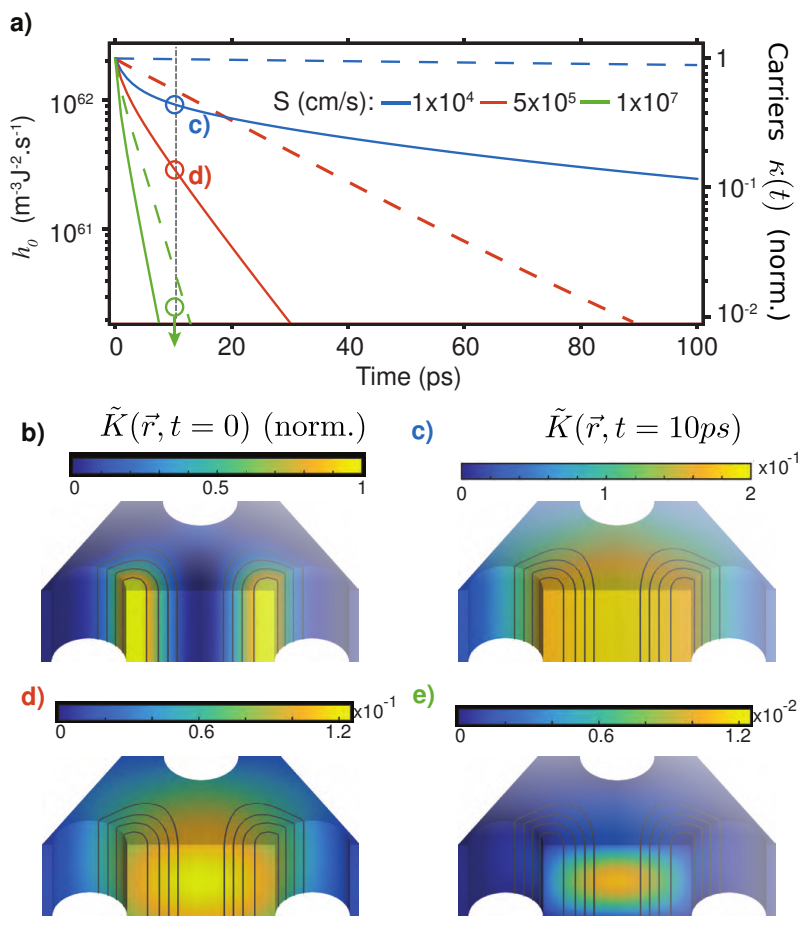

FIG. 4. $\mathrm{H}_{0}$ cavity: a) $h_{0}(t)$ (solid, left axis) and spatially averaged kernel function $\kappa(t)$ (dashed, right axis), calculated for different values of the surface recombination velocity $S$. Note that the initial value is independent on the diffusion parameters. Normalized spatial distribution $\tilde{K}(\boldsymbol{r}, t)$ of the carriers at excitation $\left(t=0^{+}\right)$b) and after $10 \mathrm{ps}$ (denoted by the dashed line in panel a), with $\left.S=10^{4} \mathrm{~cm} \cdot \mathrm{s}^{-1} \mathrm{c}\right), S=$ $5 \times 10^{5} \mathrm{~cm} \cdot \mathrm{s}^{-1} \mathrm{~d}$ ) and $S=1 \times 10^{7} \mathrm{~cm} \cdot \mathrm{s}^{-1} \mathrm{e}$ ).

GaAs) and in one case followed by surface passivation through Atomic Layer Deposition (ALD) [11]. The recovery dynamics is measured by a non-degenerate pumpprobe technique. The nonlinear resonators (Fig. 3) have been designed to provide two resonances spaced by about $10 \mathrm{~nm}$, located in the C-band of the Telecom spectrum (about $1550 \mathrm{~nm}$ ) and with loaded Q factor between 2000 and 3000. The resonator results from the coupling of two $\mathrm{H}_{0}$ cavities so that the two resonances are associated to the symmetric and anti-symmetric combination of the fundamental modes of the $\mathrm{H}_{0}$ resonators. As a consequence, the field intensity of these two modes overlaps nearly perfectly and this maximizes the nonlinear coupling. Thus, the excitation of either modes induces nearly the same spectral shift for both resonances.

The pump-probe measurement is based on a femtosecond mode-locked laser (MLL) at a repetition rate of $36 \mathrm{MHz}$ (Optisiv). As detailed in ref. [11], the pump is obtained by spectral slicing and amplification, resulting into a 6 ps pulse. The pump is set with a small detuning (roughly $0.25 \mathrm{~nm}$ on the blue side) from the high energy resonance (Fig. 5). The full spectrum of the laser, attenuated, is used as a "white light" probe. The transmitted spectra around the low energy resonance is acquired as a 


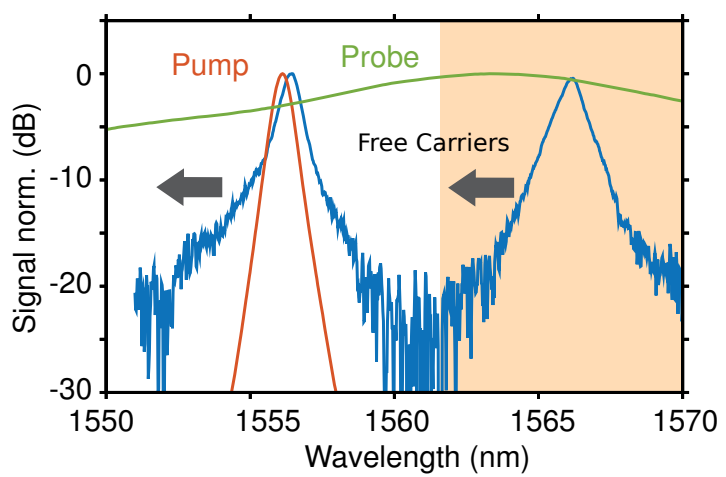

FIG. 5. Transmission (blue), pump (red) and probe (green) spectra in the pump-probe experiment. The orange shaded area represents the part of the spectrum acquired to generate the time-resolved maps in fig. 6. The arrows denote the spectral shift of both resonances resulting from by the generation of the free carriers.

function of the pump-probe delay, resulting into a spectrally and temporally resolved map (Fig. 6).

It is enlightening to compare these measured maps with their simulated counterparts, where pump and probe, as measured, are used as excitation terms. The model itself consists in eqs. 4, 6, 8, 10 and 21, which are integrated using a standard high-order adaptative routine for ordinary differential equations. The map is constructed by solving the equations for each value of the pump-probe delay and the Fourier-transforming the calculated outputs to generate the spectra.

The parameters used in the model are given in appendix D. All these parameters have been determined independently of the pump-probe measurements. They have been either calculated (e.g. the nonlinear volumes), or obtained from the literature (e.g. the properties of the semiconductor bulk material) or fitted from the linear transmission measurements (e.g. the coupling factor and the resonances), as shown in Fig. 3. This procedure singles out one parameter, the surface recombination $S$, which is process and material dependent. It is therefore a sensible option to consider it as a fitting parameter when comparing the experiment with the model. This model has been used to discuss the case of GaAs and coated GaAs PhC gates [11]. Here the discussion is generalized to a broader range of materials and geometries.

The comparison between the experimental and calculated maps in Fig. 6 reveals that not only the displacement of the resonance is well reproduced, but also other features are well captured, such as, for instance, the spectral broadening and the drop in the transmission at the pump-probe coincidence. This is true for the three cases considered here. Another important consideration is about the different response of the three devices, in terms of recovery time and extent of the spectral shift. Since the structural parameters (Q-factor and splitting of the resonances, as well as the nonlinear absorption and carrier-induced index change) are very similar (see table III), these changes are explained by the different surface recombination $S$, which therefore plays a dominant role, and not only in terms of recovery time.

A more quantitative comparison is possible by defining the normalized transmission and the frequency shift (Fig. 7) as the first order momentum of the spectrum:

$$
\Delta \nu_{c}(t)=-\nu_{r e s}+\int \Delta \nu(t, \nu) S(t, \nu) \mathrm{d} \nu
$$

As extensively discussed in ref. [11], the very large value of the surface recombination $S$ in uncoated GaAs leads to a basically instantaneous response (more precisely, faster than the duration of the excitation), also resulting into a decrease of the spectral shift. This behaviour is well reproduced when considering $S=10^{7} \mathrm{~cm} . \mathrm{s}^{-1}$, which is consistent with the literature. Coating with ALD decreases the surface recombination and favours the buildup of a larger population of free carriers, and thereby a larger spectral shift. This leads to an optimum for using these cavities as fast and still energy-efficient switches, which is the point of ref. [11].

The case of $\mathrm{InP}$ is quite different, as surface recombination velocity is even smaller, and this results into a slightly larger spectral shift but also into a much longer recovery time, as apparent in Fig. 7. In this case, it takes about 100 ps to observe an almost complete recovery of the resonance, which is much longer than in GaAs. The agreement is excellent for both the spectral shift and the transmission. The changes are explained by a change of the surface recombination velocity $S$ is $1 \times 10^{7} \mathrm{~cm} . \mathrm{s}^{-1}$ (GaAs), $3 \times 10^{5} \mathrm{~cm} . \mathrm{s}^{-1}$ (GaAs coated) and $1 \times 10^{3} \mathrm{~cm} . \mathrm{s}^{-1}$ ( $\mathrm{InP}$, which is consistent with the dominant role of diffusion and surface recombination. The discrepancy of the measured transmission with the calculations is due to the spectral features in the transmission of the output waveguide. These are the consequences of disorderinduced scattering [39], primarily affecting the output waveguide, which, in the InP sample is much longer. The other samples have been designed with short input and output waveguides and therefore show a clean double peaked line-shape (Fig. 3). We note that in the conditions of these experiments, carrier induced nonlinearity is by far dominating the Kerr contribution. Shorter pulses might evidence a greater role of the Kerr effect, as observed in GaAs micropillars [40].

We consider that our model is accurate enough to capture the response of semiconductor photonic crystal cavities.

\section{IMPACT OF THE GEOMETRY ON THE DYNAMICS}

The Shockley formula provides a rough estimate of the effective carrier lifetime in sub-wavelength resonators, by assuming an average distance from the surface. The model introduced here takes the geometry of the structure into account accurately, as it is based on solving the 


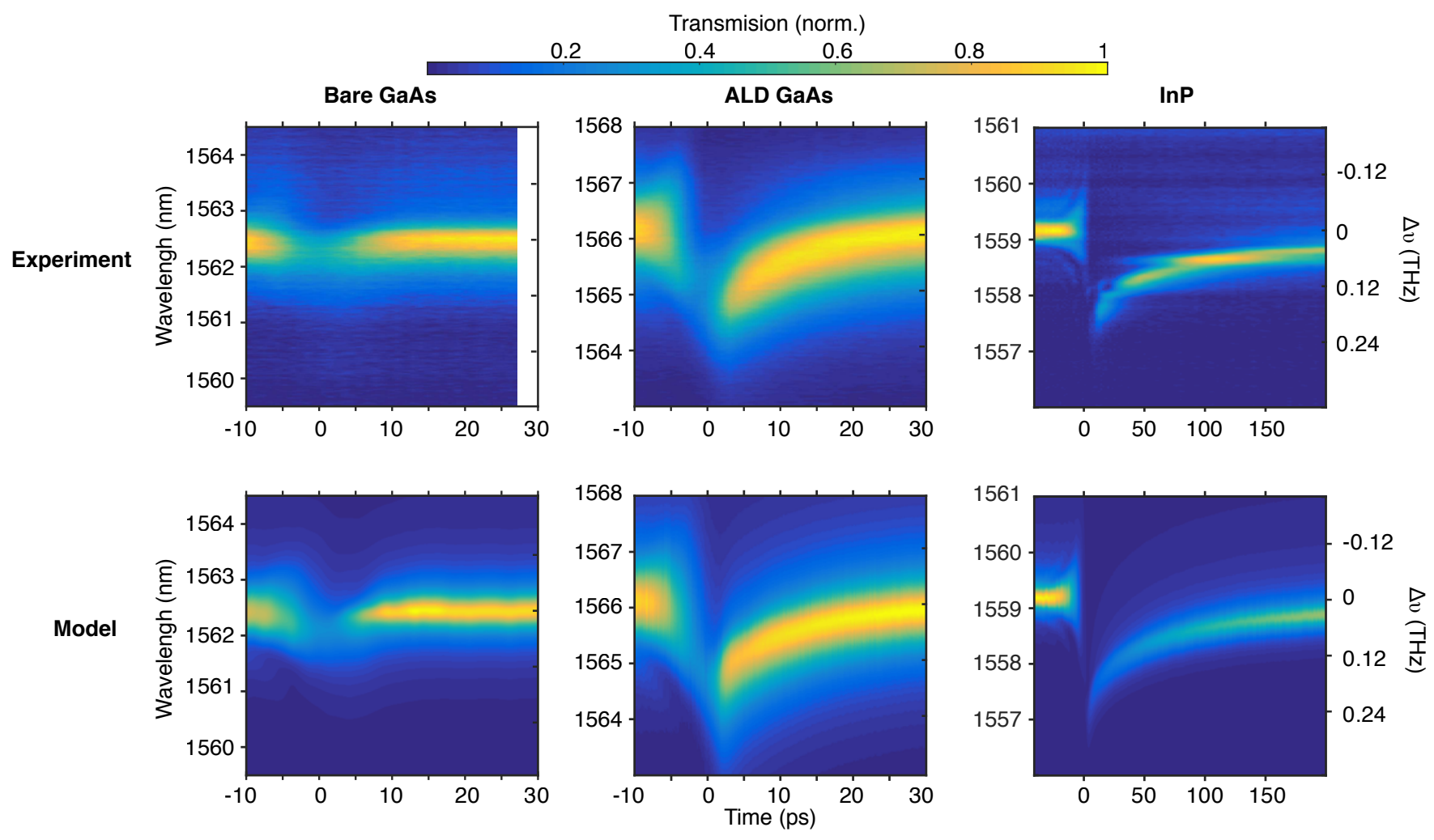

FIG. 6. Experimental (up row) and calculated (bottom row) spectrally and temporally resolved pump-probe maps. Columns, from left to right, relate to InP, bare GaAs and ALD- coated GaAs.

ambipolar diffusion equation exactly. Thus we apply our model to single out the role of the geometry in determining the nonlinear dynamical response.

Different cavities are considered here, all widely used, namely: $\mathrm{H}_{0}$ (ref. [23] and Fig. 8a), $\mathrm{H}_{1}$ (ref.[41] and Fig. 8b) $\mathrm{L}_{3}$ (ref. [42] and Fig. 8c) and $\mathrm{L}_{5}$ (ref. [42] and Fig. 8d). In order to highlight the role of the geometry, the coupled $\mathrm{Q}$ factor is set to the same value, namely $Q=3000$. What instead is different is the volume of the optical mode, more specifically $V_{\chi}$, which primarily impacts the level of optical power required for triggering enough nonlinear absorption, and the carrier dynamics, i.e. the $h_{0}$ function, which both affects the speed and the amplitude of the nonlinear response.

Here it is apparent that geometry is extremely important, for instance, $V_{\chi}$ differs by about an factor two between $\mathrm{L}_{3}$ and $\mathrm{H}_{0}$, and $h_{0}(0)$ is about one order of magnitude larger in the latter (see table I). Thus, an order of magnitude improvement in energy efficiency can be ascribed to the cavity design. Also, the $h_{0}(0)$ can be used for a quick estimate of the maximum of the carrier density. For instance, let us consider that the field in the cavity is such that it contains $W=100 \mathrm{fJ}$ and let us consider a lifetime of $\tau_{c}=10 \mathrm{ps}$, then with $h_{0}(0)=3.5 \times 10^{62} \mathrm{~J}^{-2} \mathrm{~s}^{-1} \mathrm{~m}^{-3}$ we obtain $N=10^{18} \mathrm{~cm}^{-3}$.

The impact of geometry on the time response is less straightforward. It is interesting to first consider the ini-
TABLE I. Cavity modal volume $V_{\text {mod }}$, nonlinear modal volumes $V_{\chi}, V_{3 P A}$ and maximum of the effective carrier density response function, for InP and GaAs (ALD).

\begin{tabular}{llcccc}
\hline \hline Design & $\begin{array}{l}V_{\text {mod }} \\
\times 10^{-18} \mathrm{~m}^{-3}\end{array}$ & $V_{\chi}$ & $V_{3 P A}$ & $\begin{array}{c}h_{0}(0)(\mathrm{InP}) \\
\times 10^{61} \mathrm{~J}^{-2} \mathrm{~s}^{-1} \mathrm{~m}^{-3}\end{array}$ & $(\mathrm{GaAs})$ \\
\hline $\mathrm{L}_{5}$ & 0.08 & 4 & 0.88 & 6.7 & 4.6 \\
$\mathrm{~L}_{3}$ & 0.057 & 3 & 0.67 & 12 & 8.1 \\
$\mathrm{H}_{1}$ & 0.064 & 2.6 & 0.60 & 14 & 10 \\
$\mathrm{H}_{0}$ & 0.045 & 2.2 & 0.32 & 51 & 35 \\
\hline \hline
\end{tabular}

tial spatial distribution of the photo-generated carriers, which is directly related to the distribution of the electric field of the optical mode $|\boldsymbol{U}|^{4}$. This is shown in Fig. 8, subpanels a-d. It is apparent that in the case of cavities $\mathrm{H}_{0}$ and $\mathrm{H}_{1}$ carriers are generated close to the holes, where they are likely to recombine, whereas in panel c-d it is apparent that they need to diffuse away, before reaching the holes.

This explains in part the different decay of the effective density $N_{\text {eff }}$, more precisely $h_{0}(t)$, calculated in Fig. 8e,f. Let first consider the short time scale, and the case of low surface recombination (e.g. InP). It has been first pointed out [18] that an initial phase of fast decay due to diffusion is followed by a slower dynamics. What is interesting here is the role played by the geometry. 
Bare GaAs
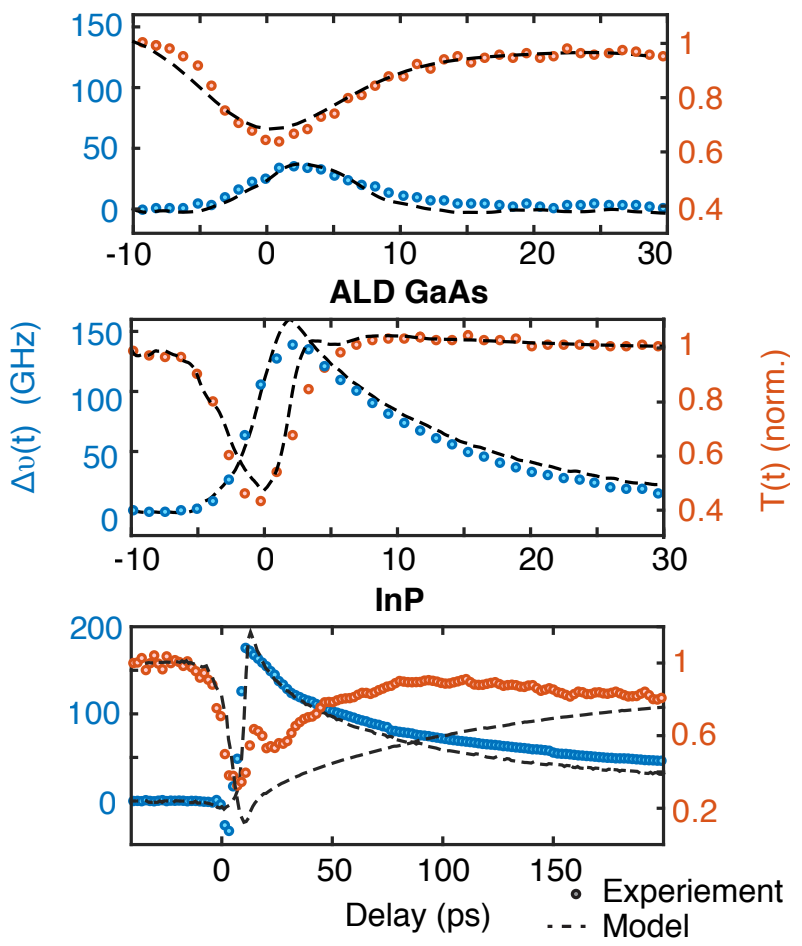

FIG. 7. Measured (circles) and calculated (dashed lines) spectral shift (blue) and transmission (red) for a) bare GaAs, b) ALD coated GaAs and c) InP. The corresponding surface recombination velocity is $S=1 \times 10^{7} \mathrm{~cm} . \mathrm{s}^{-1}, 3 \times 10^{5} \mathrm{~cm} . \mathrm{s}^{-1}$ and $1 \times 10^{3} \mathrm{~cm} \cdot \mathrm{s}^{-1}$, respectively.

The decay of the effective density in the $\mathrm{H}_{0}$ cavity is way faster, but at least at the very first stage, the $\mathrm{H}_{1}$ resonator is almost as fast. This is mostly because the initial distribution of photogenerated carriers is much more concentrated in the $\mathrm{H}_{0,1}$ cavities than in the others. After this initial stage, the dynamics of the $\mathrm{H}_{1}$ slows down and tends to approach the behaviour of the $\mathrm{L}_{3}$ and $\mathrm{L}_{5}$ structures. Actually, in these two cavities carriers diffuse mostly perprendicular to the cavity main axis, owing to a larger concentration gradient. This explains why the decay of the effective density is almost identical for the two. A few picoseconds after the excitation, the carriers accumulate with a spatial distribution which is roughly Gaussian and extending to the geometric volume of the cavity.

When the surface recombination increases, then the geometry tends to be less important. Consider the case of GaAs with coated surface $\left(S=3 \times 10^{5} \mathrm{~cm} / \mathrm{s}\right)$, shown in Fig. 8f. Here, the $\mathrm{H}_{0}$ is still about twice as fast as the other cavities, but the difference is much less striking. This is consistent with the picture of surface recombination eventually dominating the dynamics.

The calculated spectral shift $\Delta \nu$, which is obtained by solution of the full model, is shown in Fig. 9. Here the temporal response is also determined by the optical prop-
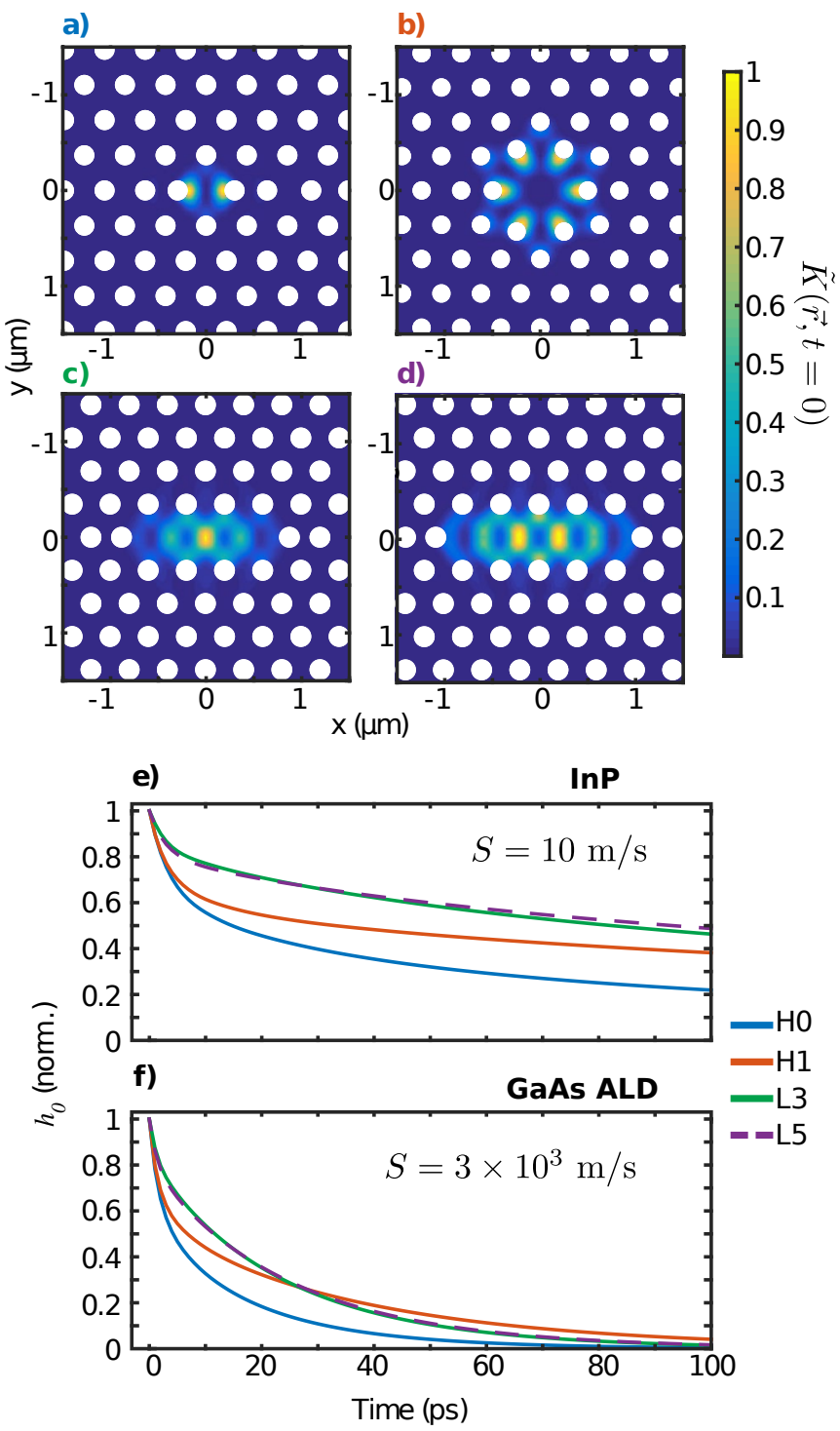

FIG. 8. Initial distribution of free carriers $\tilde{K}(\boldsymbol{r} ; 0)$ calculated for a) $\mathrm{H}_{0}$ b) $\mathrm{H}_{1}$ c) $\mathrm{L}_{3}$ and d) $\mathrm{L}_{5}$ design. Decay of the carrier effective response function, normalized: $h_{0}(t) / h_{0}(0)$, for GaAs coated with ALD and InP.

erties of the resonator (the cavity lifetime) and by the excitation. Still, given the loaded $\mathrm{Q}$ factor of the resonators $(\approx 3000$, hence cavity lifetime $Q / \omega \approx 3 \mathrm{ps})$ and the duration of the pulses, also in the same range, the decay is clearly dominated by the carrier dynamics. What is clearly visible here is the role of the nonlinear volume $V_{\chi}$ on the amount of pulse energy required to achieve a prescribed spectral shift (here $250 \mathrm{GHz}$ ), again confirming the net advantage of the $\mathrm{H}_{0}$ cavity. The difference is substantial but not as large as Table I might suggest. 


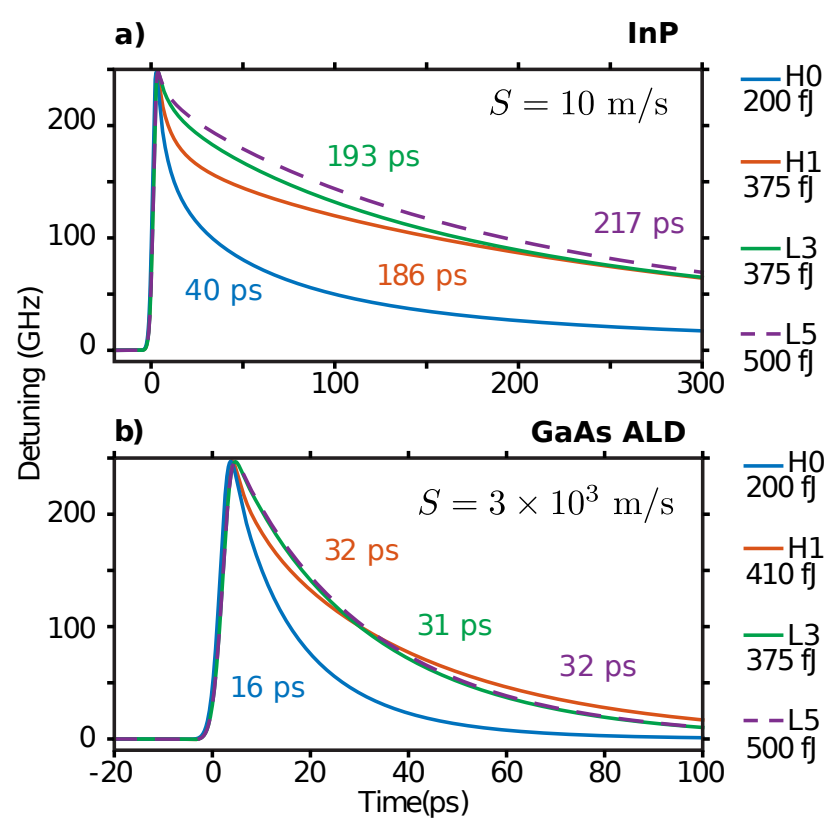

FIG. 9. Calculated spectral shift for a) InP and b) ALD coated GaAs, corresponding to the geometries in fig. 8. The coloured text represents a tentative exponential fit of the decay.

\section{LINEAR ABSORPTION}

Our model allows the comparison of optical switches activated either by linear or nonlinear absorption. The peculiarity of nonlinear absorption is that the material used for the nonlinear resonator can also be used to create the access waveguides. In fact, at operating power levels, the material is transparent except in the cavity, owing to the enhanced strength of the optical field. Conversely, in optical microcavities, nonlinear absorption can be a rather energy-efficient process, in striking contrast with macroscopic cavities, where nonlinear absorption is usually considered as a perturbation.

Also, strong linear absorption spoils the Q factor of the cavity, thus, a weak linear absorption is preferable. The most efficient microcavity all-optical switch resulted from by the optimisation of the material absorption by choosing the suitable composition of the GaInAsP alloy, such that it entails a weak absorption [6]. Moreover, the nonlinear index change is maximized owing to the bandfilling contribution [28].

This situation is considered in our model by introducing a modified version of eqs. 12, 17, 18, 20, 21 and 23,

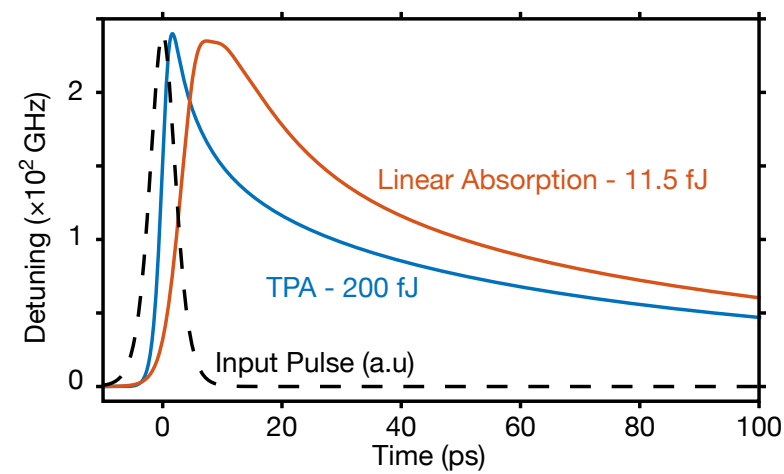

FIG. 10. Spectral shift in a cavity induced by TPA (blue line) or by linear absorption. The input excitation is also represented (dashed line).

namely:

$$
\begin{aligned}
& G(\boldsymbol{r})=\alpha \frac{c_{0} n \varepsilon_{0}}{2 \hbar \omega}|\boldsymbol{U}(\boldsymbol{r})|^{2}|A|^{2} \\
& N(\boldsymbol{r}, t)=\int_{-\infty}^{t} \tilde{K}(\boldsymbol{r} ; t-\tau)|A(\tau)|^{2} \mathrm{~d} \tau \\
& \tilde{K}(\boldsymbol{r} ; \tau)=\alpha \frac{c_{0} \varepsilon_{0}}{2 \hbar \omega} \varepsilon_{r} \int_{V} K\left(\boldsymbol{r}, \boldsymbol{r}^{\prime} ; \tau\right)|\boldsymbol{U}(\boldsymbol{r})|^{2} \mathrm{~d} \boldsymbol{r}^{\prime} \\
& N(\boldsymbol{r}, t)=\tilde{K}(\boldsymbol{r}, t)\left|A_{0}\right|^{2} \Delta t \\
& N_{e f f}(t)=\int_{-\infty}^{t} h_{0}(t-\tau)|A(\tau)|^{2} \mathrm{~d} \tau \\
& h_{0}\left(t_{0^{+}}\right)=\frac{\alpha c_{0} \varepsilon_{0}^{2}}{4 \hbar \omega} \int_{V} \varepsilon_{r}|\boldsymbol{U}(\boldsymbol{r})|^{4} \mathrm{~d} V=\frac{\alpha c_{0}}{\hbar \omega V_{\chi}}
\end{aligned}
$$

We consider two structurally identical devices, aiming at showing that linear and nonlinear absorption induce a different nonlinear dynamics in the cavity. In order to single this contribution out, we have used the same parameters as for the InP sample discussed in sect. V. The sample labelled as "Linear absorption" is made of GaInAsP, as in ref. [6]. The relevant parameters are in table IV, however, the surface recombination is arbitrarily taken as that of InP, in order to single out the role of the geometry and the diffusion process, in addition to the absorption mechanism.

The result is shown in Fig. 10. The excitation energy is adjusted to reach the same spectral shift, again 250 GHz. As expected, the energy is about a factor 20 lower in the case of the optimized material. It is however intriguing to note a striking difference in the dynamics. First, the leading edge of $\delta \nu$ is much steeper in the case of the nonlinear absorption, which can be understood in the dependence on $|A|^{4}$ instead of $|A|^{2}$ of the excitation. Second, the faster initial decay, which is related to the diffusion, is largely explained by the stronger gradient of the initial spatial distribution of the photo-excited carriers, i.e. $|\boldsymbol{U}|^{4}$ instead of $|\boldsymbol{U}|^{2}$.

Note that the $\mathrm{Q}$ factor here is designed to be smaller than in ref. [6], in order to be consistent with calcula- 
tions in the previous sections of our paper. We note that TPA device is faster although the ambipolar coefficient of $\operatorname{InP}\left(D_{a}=9 \mathrm{~cm}^{2} / \mathrm{s}\right)$ is smaller than $\operatorname{InGaAsP}\left(D_{a}=17\right.$ $\left.\mathrm{cm}^{2} / \mathrm{s}\right)$. This example shows the flexibility of our model and the capability of modelling a variety of devices and materials.

\section{CONCLUSIONS}

We have introduced a model for the dynamical response of nonlinear nanoscale semiconductor resonators. The diffusion of the photoexcited carriers is taken into account rigorously, within the ambipolar approximation, using the Green's function formalism. The model is able to reproduce the experimentally measured response accurately and with a minimal set of fitting parameters. We use it to highlight the role of the geometry of the resonator and that of the linear or nonlinear generation of free carriers. This model can be used for any semiconductor nonlinear resonator and would greatly help the design of complex devices comprising of mutually coupled resonant devices. This would provide a reliable description of the resulting complex nonlinear dynamics. Also in the case of nominally independent wavelength multiplexed optical gates, such as in ref. [8], such a model might be useful in accounting for potential and unwanted nonlinear crosstalk, which is important for a correct design of complex photonic circuits.

\section{ACKNOWLEDGEMENTS}

We acknowledge Agence Nationale de la Recherche through the contracts AUCTOPUSS and ETHAN. We thank Borge Vinter for enlightening discussions.

\section{Appendix A: Approximations in the CMT}

Let us consider the simple case of one cavity coupled to a waveguide, and $|\gamma|>>\left|\Gamma_{0}\right|$. At steady state and at resonance $\omega=\omega_{k}$, eq. 2 reduces to $0=-\frac{\gamma}{2} A+\kappa S_{\text {in }}$, hence $|A|^{2}=2 \gamma^{-1}\left|S_{i n}\right|^{2}$. Moreover, the energy density in the waveguide is related to the power by the group velocity $v_{g}$ and the waveguide cross section $\sigma_{w g}$, hence the energy density in the waveguide is $W_{w g}=\frac{\left|S_{i n}\right|^{2}}{v_{g} \sigma_{w g}}=2 \frac{\gamma W_{c a v} V_{c a v}}{v_{g} \sigma_{w g}}$, thus the ratio $\frac{W_{c a v}}{W_{w g}}=\frac{v_{g} \sigma_{w g}}{2 \gamma V_{c a v}}$. Assuming the normal mode of the cavity is the stationary counterpart of the travelling waveguide modes (e.g. $\mathrm{L}_{3}, \mathrm{~L}_{5}$ cavities and line defect waveguides built out of the same lattice), then $V_{c a v} / \sigma_{w g} \approx L_{c a v}$ the waveguide length and therefore $2 \frac{V_{c a v}}{\sigma_{w g} v_{g}}$ is the cavity round-trip time $\tau_{r}$. This allows interpreting $\frac{v_{g} \sigma_{w g}}{2 \gamma V_{\text {cav }}}$ as $\gamma^{-1} \tau_{r}^{-1}=1 / F$ the inverse of the finesse of the cavity, which is a well know result. In a single-mode cavity such as $\mathrm{H}_{0}$, this number is very close to the $\mathrm{Q}$ factor.

\section{Appendix B: Calculation of the Kerr and TPA nonlinear terms}

The nonlinear interaction terms have been derived in the context of the coupled mode theory in countless publications and monographies, see e.g. [43]. For the sake of consistency with our own notation, we provide here the derivation of the nonlinear coefficients.

With the usual approximations (rotating wave, set of orthogonal normal modes $\boldsymbol{U}$ ), the nonlinear perturbation of the complex envelope reads:

$$
\partial_{t} A=-\imath \frac{\exp (\imath \omega t)}{4 \omega} \int \boldsymbol{U}^{*} \partial_{t}^{2} \boldsymbol{P}_{N L} d V
$$

The specific case of the third order susceptibility (Kerr nonlinearity) is considered here. Following Boyd [27]:

$$
\mathbf{P}_{N L} \cdot \mathbf{E}^{*}=n_{2} \varepsilon_{r} \varepsilon_{0}^{2} c_{0} \frac{2|\mathbf{E}|^{4}+|\mathbf{E} \cdot \mathbf{E}|^{2}}{3}
$$

which in the case of a single cavity mode becomes:

$$
\partial_{t} A=-\imath \frac{\omega n_{2} c_{0}}{4} \int \varepsilon_{0}^{2} \varepsilon_{r} \chi_{r} \frac{2|\mathbf{U}|^{4}+|\mathbf{U} \cdot \mathbf{U}|^{2}}{3} d V|A|^{2} A
$$

where we account for the inhomogeneity of the nonlinear tensor by introducing a factor $\chi_{r}=\frac{\chi^{(3)}(\boldsymbol{r})}{\chi_{\text {max }}^{(3)}}$, which vanishes outside the nonlinear material. This equation is rewritten as:

$$
\partial_{t} A=\imath \Delta \omega(A) A=-|A|^{2} V_{\chi}^{-1} c_{0} n_{2} \omega
$$

This defines the instantaneous frequency shift and the Kerr volume:

$$
V_{\chi}^{-1}=\frac{\varepsilon_{0}^{2}}{4} \int \varepsilon_{r} \chi_{r} \frac{2|\mathbf{U}|^{4}+|\mathbf{U} \cdot \mathbf{U}|^{2}}{3} d V
$$

The two photon absorption is related to the Kerr effect, and is treated accordingly. Thus eq. 12 is generalized by replacing $|\boldsymbol{U}|^{4}$ with $\frac{2|\mathbf{U}|^{4}+|\mathbf{U} \cdot \mathbf{U}|^{2}}{3}$. This leads to a correction to eq. 12 :

$$
G(\boldsymbol{r})=\beta_{T P A} \frac{c_{0}^{2} \varepsilon_{0}^{2}}{8 \hbar \omega} \varepsilon_{r} \frac{2|\mathbf{U}|^{4}+|\mathbf{U} \cdot \mathbf{U}|^{2}}{3}|A|^{4}
$$

In the geometries considered here, the scalar approximation of $V_{\chi}$ is reasonably accurate, the discrepancy being about a few percent or less, and, therefore, can be safely used.

\section{Appendix C: Approximations in modelling the carriers}

Depending on the time scale considered, the dynamics of carriers is described by models of increasing complexity. The main assumption here is that the excitation is never shorted than 1 ps. Consequently, it is expected 
that excited carriers have already relaxed to their quasiequilibrium distribution, which is described by quasiFermi levels. Thus, effects of carrier heating are ignored, and the energy released by thermalized carriers, namely $2 \hbar \omega-E_{g}$ is considered as heat. We also consider that the injected power is not large enough to induce the saturation of the absorption, which is reasonable since large enough spectral shift is achieved without reaching carrier density above $10^{18} \mathrm{~cm}^{-3}$.

Recombination here can be treated with a single term, accounting for the dominant mechanism, namely nonradiative recombination at the surface. Recombination in the bulk of the material is orders of magnitude lower, also radiative and Auger recombination are safely negligible. The effective carrier density $N_{\text {eff }}$ is introduced to relate the calculation of the diffusion equation to the spectral shift of the resonance. The perturbation analysis is used to connect the change of the dielectric permittivity $\Delta \varepsilon$ to the spectral shift $\Delta \omega$ :

$$
\Delta\left(\omega^{2}\right)=-\omega^{2} \frac{\int \varepsilon_{0} \Delta \varepsilon_{r}|\mathbf{U}|^{2} \mathrm{~d} V}{\int \varepsilon_{0} \varepsilon_{r}|\mathbf{U}|^{2} \mathrm{~d} V}
$$

Hence: $\Delta \omega=-\frac{\omega}{4} \int \varepsilon_{0} \Delta \varepsilon_{r}|\mathbf{U}|^{2} \mathrm{~d} V$. Moreover $\Delta \varepsilon_{r}=$ $2 n \Delta n=2 n\left(\partial_{N} n+\partial_{P} n\right)$, which, in the ambipolar approx- imation $N=P$ becomes $\partial_{N_{a}} n=\partial_{N} n+\partial_{P} n$. Finally:

$$
\Delta \omega=-\frac{\omega}{2} n \varepsilon_{0} \frac{\partial n}{\partial N_{a}} \int N|\mathbf{U}|^{2} \mathrm{~d} V
$$

Consider a volume with uniform carrier density $N(\boldsymbol{r})=$ $N_{\text {eff }}$ and $\varepsilon_{r}$. Then eq. C2 becomes:

$$
\Delta \omega=-\frac{\omega}{n} \frac{\partial n}{\partial N} N_{e f f}
$$

Comparing (C2) and (C3) leads to the definition of the effective carrier density:

$$
N_{e f f}=\int N \frac{\varepsilon_{0} \varepsilon_{r}|\mathbf{U}|^{2}}{2} \mathrm{~d} V
$$

\section{Appendix D: Tables}

TABLE II. Model, summarized

\begin{tabular}{lr}
\hline \hline Field Dynamics & $\dot{\mathbf{A}}=\mathbf{M ~ A}-\mathbf{R S ~ S}_{\text {in }}$ \\
Kerr NL & $\imath \Delta \omega_{k}-\frac{\Delta \Gamma_{k}}{2}=\left|A_{k}\right|^{2} c_{0}\left(\imath \omega n_{2}-\beta_{T P A} \frac{c_{0}}{2}\right) V_{\chi}^{-1}$ \\
Free Carriers NL & $\imath \Delta \omega_{k}-\frac{\Delta \Gamma_{k}}{2}=\left.\imath \frac{\omega}{n} \frac{\partial \tilde{n}}{\partial N}\right|_{F C} N_{e f f}^{(k)}$ \\
Free Carriers & $N_{e f f}(t)=\int_{-\infty}^{t} h_{0}(t-\tau)|A(\tau)|^{4} \mathrm{~d} \tau$ \\
Dynamics & \\
\hline
\end{tabular}

[1] A. E. Willner, S. Khaleghi, M. R. Chitgarha, and O. F. Yilmaz, Journal of Lightwave Technology 32, 660 (2014).

[2] J. L. Jewell, S. L. McCall, A. Scherer, H. H. Houh, N. A. Whitaker, A. C. Gossard, and J. H. English, Applied Physics Letters 55, 22 (1989).

[3] V. Van, T. A. Ibrahim, P. P. Absil, F. G. Johnson, R. Grover, and P.-T. Ho, Selected Topics in Quantum Electronics, IEEE Journal of 8, 705 (2002).

[4] V. R. Almeida, C. A. Barrios, R. R. Panepucci, and M. Lipson, Nature 431, 1081 (2004).

[5] T. Tanabe, M. Notomi, S. Mitsugi, A. Shinya, and E. Kuramochi, Appl. Phys. Lett. 87, 151112 (2005).

[6] K. Nozaki, T. Tanabe, A. Shinya, S. Matsuo, T. Sato, H. Taniyama, and M. Notomi, Nature Photon. 4, 477 (2010).

[7] K. Nozaki, A. Shinya, S. Matsuo, Y. Suzaki, T. Segawa, T. Sato, Y. Kawaguchi, R. Takahashi, and M. Notomi, Nature Photonics 6, 248 (2012).

[8] K. Nozaki, E. Kuramochi, A. Shinya, and M. Notomi, Optics Express 22, 14263 (2014).

[9] Y. Yu, H. Hu, L. K. Oxenlwe, K. Yvind, and J. Mork, Optics Letters 40, 2357 (2015).

[10] A. Bazin, K. Lenglé, M. Gay, P. Monnier, L. Bramerie, R. Braive, G. Beaudoin, I. Sagnes, and F. Raineri, Appl. Phys. Lett. 104, 011102 (2014).

[11] G. Moille, S. Combrié, L. Morgenroth, G. Lehoucq, F. Neuilly, B. Hu, D. Decoster, and A. de Rossi, Laser
Photon. Rev, n/a (2016).

[12] H. Thyrrestrup, E. Yüce, G. Ctistis, J. Claudon, W. L. Vos, and J.-M. Grard, Applied Physics Letters 105, 111115 (2014).

[13] A. M. Yacomotti, P. Monnier, F. Raineri, B. B. Bakir, C. Seassal, R. Raj, and J. A. Levenson, Physical Review Letters 97 (2006), 10.1103/PhysRevLett.97.143904.

[14] S. Malaguti, G. Bellanca, A. de Rossi, S. Combrié, and S. Trillo, Physical Review A 83, 051802 (2011).

[15] N. Cazier, X. Checoury, L.-D. Haret, and P. Boucaud, Optics express 21, 13626 (2013).

[16] Y. Dumeige and P. Fron, Physical Review A 84 (2011), 10.1103/PhysRevA.84.043847.

[17] P. Colman, P. Lunnemann, Y. Yu, and J. Mrk, arXiv preprint arXiv:1604.06215 (2016).

[18] T. Tanabe, H. Taniyama, and M. Notomi, J. Lightwave Technol. 26, 1396 (2008).

[19] C. Manolatou, M. J. Khan, S. Fan, P. R. Villeneuve, H. A. Haus, and J. D. Joannopoulos, IEEE J. Quantum Electron. 35, 1322 (1999).

[20] G. Lecamp, J.-P. Hugonin, and P. Lalanne, Optics express 15, 11042 (2007).

[21] P. T. Kristensen and S. Hughes, ACS Photonics 1, 2 (2014).

[22] J. Lian, S. Sokolov, E. Yüce, S. Combrié, A. de Rossi, and A. P. Mosk, Opt. Lett. 40, 4488 (2015).

[23] Z. Zhang and M. Qiu, Opt. Exp. 12, 3988 (2004). 
TABLE III. Simulation Parameters value used in the CMT model for bare GaAs, ALD-coated GaAs and InP (c.f section $\mathrm{V}$ )

\begin{tabular}{lcccl}
\hline \hline Param. & Bare GaAs & ALD GaAs & InP & Units \\
\hline$\omega_{1}$ & 192.31 & 191.89 & 192.85 & $\times 2 \pi \mathrm{THz}$ \\
$\omega_{2}$ & 192.50 & 192.13 & 192.85 & $\times 2 \pi \mathrm{THz}$ \\
$\mu$ & 3.3059 & 3.6581 & 3.5853 & $\times 10^{12} \mathrm{~s}^{-1}$ \\
$\kappa_{1}^{a}$ & 5.79 & 7.76 & 6.36 & $\times 10^{5} \mathrm{~s}^{-1 / 2}$ \\
$\kappa_{2}^{a}$ & 0 & 0 & 0 & $\times 10^{5} \mathrm{~s}^{-1 / 2}$ \\
$\kappa_{1}^{b}$ & 5.79 & 7.76 & 6.355 & $\times 10^{5} \mathrm{~s}^{-1 / 2}$ \\
$\kappa_{2}^{b}$ & 0 & 0 & 3.759 & $\times 10^{5} \mathrm{~s}^{-1 / 2}$ \\
$\Gamma_{0,1}$ & 1.21 & 1.21 & 1.21 & $\times 10^{-12} \mathrm{~s}^{-1}$ \\
$\Gamma_{0,2}$ & 1.21 & 1.21 & 1.21 & $\times 10^{-12} \mathrm{~s}^{-1}$ \\
$S$ & $1 \times 10^{7}$ & $3.3 \times 10^{5}$ & $1 \times 10^{3}$ & $\mathrm{~cm} . \mathrm{s}^{-1}$ \\
$E_{p m p}$ & 100 & 150 & 500 & $\mathrm{fJ}$ \\
$E_{p r b}$ & 1 & 1 & 1 & $\mathrm{fJ}$ \\
$\omega_{p m p}$ & 192.99 & 192.66 & 193.38 & $\times 2 \pi \mathrm{THz}$ \\
$\omega_{p r b}$ & 191.93 & 191.39 & 192.30 & $\times 2 \pi \mathrm{THz}$ \\
$t_{p m p}$ & 5 & 5 & 6 & $\mathrm{ps}$ \\
$t_{p r b}$ & $<0.5$ & $<0.5$ & 1 & $\mathrm{ps}$ \\
\hline \hline
\end{tabular}

[24] S. Combrié, G. Lehoucq, A. Junay, S. Malaguti, G. Bellanca, S. Trillo, L. Ménager, J. P. Reithmaier, and A. de Rossi, Appl. Phys. Lett. 103, 193510 (2013).

[25] A. de Rossi, M. Lauritano, S. Combrié, Q. V. Tran, and C. Husko, Phys. Rev. A 79, 043818 (2009).

[26] Thermal response can be included in the model, by introducing an auxialiary dynamic equation. This is useful when analysing long-term response or situation with very fast heating.

[27] R. W. Boyd, Nonlinear Optics (Academic Press, 2013).

[28] B. R. Bennett, R. A. Soref, and J. A. Del Alamo, IEEE J. Quantum Electron. 26, 113 (1990).

[29] J. Mark and J. Mork, Applied Physics Letters 61, 2281 (1992).

[30] S. M. Sze and K. K. Ng, Physics of Semiconductor Devices (John Wiley \& Sons, Inc., 1981).

[31] W. Shockley, Phys. Today 3, 16 (1950).
[32] C. Husko, A. de Rossi, S. Combrié, Q. V. Tran, F. Raineri, and C. W. Wong, Appl. Phys. Lett. 94, 021111 (2009).

[33] D. D. Nolte, Solid-State Electron. 33, 295 (1990).

[34] T. Tanabe, K. Nishiguchi, A. Shinya, E. Kuramochi, H. Inokawa, M. Notomi, K. Yamada, T. Tsuchizawa, T. Watanabe, H. Fukuda, H. Shinojima, and S. Itabashi, Appl. Phys. Lett. 90, 031115 (2007).

[35] M. Heuck, S. Combrié, G. Lehoucq, S. Malaguti, G. Bellanca, S. Trillo, P. T. Kristensen, J. Mork, J. P. Reithmaier, and A. de Rossi, Appl. Phys. Lett. 103, 181120 (2013).

[36] Y. Yu, E. Palushani, M. Heuck, N. Kuznetsova, P. T. Kristensen, S. Ek, D. Vukovic, C. Peucheret, L. K. Oxenløwe, S. Combrié, A. de Rossi, K. Yvind, and J. Mørk, Opt. Exp. 21, 31047 (2013).

[37] R. Bose, J. S. Pelc, S. Vo, C. M. Santori, and R. G. Beausoleil, Optics Express 23, 12732 (2015).

[38] A. D. Bristow, J.-P. R. Wells, W. H. Fan, A. M. Fox, M. S. Skolnick, D. M. Whittaker, A. Tahraoui, T. F. Krauss, and J. S. Roberts, Applied Physics Letters 83, 851 (2003).

[39] M. Patterson, S. Hughes, S. Combrié, N. V. Q. Tran, A. de Rossi, R. Gabet, and Y. Jaouen, Phys. Rev. Lett. 102, 253903 (2009).

[40] E. Yüce, G. Ctistis, J. Claudon, E. Dupuy, K. J. Boller, J.-M. Grard, and W. L. Vos, JOSA B 29, 2630 (2012).

[41] M. Shirane, S. Kono, J. Ushida, S. Ohkouchi, N. Ikeda, Y. Sugimoto, and A. Tomita, J. Appl. Phys 101, 073107 (2007).

[42] Y. Akahane, T. Asano, B.-S. Song, and S. Noda, Nature 425, 944 (2003).

[43] J. D. Joannopoulos and S. G. Johnson, Photonic Crystals (Springer Science \& Business Media, 2001).

[44] M. Dinu, F. Quochi, and H. Garcia, Appl. Phys. Lett. 82, 2954 (2003).

[45] L. P. Gonzalez, J. M. Murray, and S. Krishnamurthy, Opt. Exp. 17, 8741 (2009).

[46] M. Levinshtein, S. Rumyantsev, and M. Shur, Handbook Series On Semiconductor Parameters, Vol. 2 (1996). 
TABLE IV. Parameters for GaAs, InP and InGaAsP at $\lambda=1.55 \mu m$

\begin{tabular}{|c|c|c|c|c|c|c|c|c|}
\hline Parameters & Symbol & GaAs & Ref. & $\mathrm{InP}$ & Ref. & InGaAsP & Ref. & Units \\
\hline TPA coeff. & $\beta_{T P A}$ & 10.2 & {$[44]$} & 15 & {$[45]$} & 65 & {$[6]$} & $\mathrm{cm} \cdot \mathrm{GW}^{-1}$ \\
\hline Absorption & $\alpha$ & - & - & - & - & 3 & {$[6]$} & $\mathrm{cm}^{-1}$ \\
\hline Kerr coeff. & $n_{2 I}$ & $1.6 \times 10^{-4}$ & {$[44]$} & $4.4 \times 10^{-5}$ & {$[27]$} & $3.2 \times 10^{-3}$ & {$[6]$} & $\mathrm{cm}^{2} \cdot \mathrm{GW}^{-1}$ \\
\hline Band Filling & $\left(\frac{\partial n}{\partial N_{e f f}}\right)_{R E}$ & $-2.4 \times 10^{-21}$ & {$[28]$} & $-3.5 \times 10^{-21}$ & {$[28]$} & $-8.2 \times 10^{-20}$ & {$[6]$} & $\mathrm{cm}^{3}$ \\
\hline FCA & $\frac{\partial \Gamma}{\partial N_{e f f}}$ & $3.2 \times 10^{-7}$ & {$[28]$} & $1.1 \times 10^{-6}$ & {$[28]$} & - & & $\mathrm{cm}^{3} \cdot \mathrm{s}^{-1}$ \\
\hline
\end{tabular}

